\title{
Propiedades psicométricas de la Escala de satisfacción laboral de Warr, Cook y Wall, versión en español
}

\author{
Psychometric properties of the job satisfaction scale of Warr, Cook and Wall
}

\begin{abstract}
Alicia Boluarte ${ }^{1, \text { a }}$
RESUMEN

Objetivos: Conocer las propiedades psicométricas de la Escala de Satisfacción Laboral de Warr, Cook y Wall, versión en español, en trabajadores de una institución especializada de rehabilitación. Material y métodos: Estudio de tipo psicométrico. Se incluyeron 88 trabajadores a quienes se les aplicó la Escala de Satisfacción General de Warr, Cook y Wall traducida al español y adaptada por Pérez-Bilbao y Fidalgo, compuesta por 15 ítems y 2 subdimensiones: satisfacción intrínseca y extrínseca. Se realizó análisis factorial exploratorio mediante el método de ejes principales. Resultados: los resultados demuestran que la escala de Satisfacción General de Warr, Cook y Wall es válida y confiable para una sola dimensión. Conclusiones: La valoración de la escala de satisfacción general de Warr, Cook y Wall puede hacerse a 3 niveles: general, satisfacción intrínseca y extrínseca. Sin embargo, los resultados demuestran una mayor consistencia con la medida de la satisfacción general.
\end{abstract}

PALABRAS CLAVE: Satisfacción laboral, reproductibilidad de resultados, validez de la prueba, análisis factorial. (Fuente: DeCS Bireme)

\section{SUMMARY}

Objectives: To know the psychometric properties of a general satisfaction scale in workers of specialized rehabilitation institution. Methods: Psychometric study. It included 88 workers who were applied the General Satisfaction Scale of Warr, Cook and Wall (1979) translated and adapted by Pérez-Fidalgo and Bilbao (1995), composed of 15 items and 2 subdomains : intrinsic and extrinsic satisfaction. Results: The results show that the General Satisfaction scale Warr, Cook and Wall is valid and reliable for a single dimension. Conclusions: The assessment of the scale of overall satisfaction Warr, Cook and Wall (1979) can be done at 3 levels : general, intrinsic and extrinsic satisfaction. However, the results show a major consistency with the measure of overall satisfaction.

KEYWORDS: Job satisfaction, reproducibility of results, validity. (Source: MeSH NLM)

\section{INTRODUCCIÓN}

La satisfacción laboral (SL) es vista como el conjunto de actitudes que se tienen ante el trabajo, considerándose lo que el sujeto piensa acerca de él y las actitudes y sentimientos que se generan a partir de ello (1). Para una organización es importante conocer estas actitudes con el fin de corregir malas situaciones y mejorar la satisfacción de su personal.

Los miembros de la organización pasan una parte sustancial de sus vidas en el trabajo, y muchas veces son quienes mantienen contacto directo con los usuarios, influyendo en la calidad del servicio 
brindado. De esta manera y desde una perspectiva de desarrollo organizacional, el estudio de la SL es de suma importancia en el campo de la salud pública al enfatizar en el equilibrio que ha de haber entre cada una de las partes (2).

A nivel mundial existe una diversidad de propuestas para medir la SL tanto en ámbitos públicos como privados. Meliá y Peiró (3), diseñaron y validaron un cuestionario de 5 dimensiones, en una muestra de 155 sujetos miembros formales de organizaciones laborales. Asimismo, se reportan estudios con el uso de la escala de Warr et al (4), pero solo con 10 ítems, como el estudio de Hills y col (5), en 9900 médicos australianos que trabajaban en el sector público, privado o en ambos y el estudio de Goetz y col (6), en médicos generales y personal no médico.

La utilidad de la escala general de satisfacción obedece a la brevedad y fácil administración; con fines de una detección gruesa de la satisfacción laboral. Sin embargo, la carencia de instrumentos de medición apropiados para muestras procedentes de salud pública no permite valorar con exactitud, los factores organizacionales, lo que posterga el proceso de modernización del Estado.
Ante ello, se realizó el estudio con el objetivo de evaluar las propiedades psicométricas de la Escala de satisfacción laboral de Warr, Cook y Wall, versión en español (Escala General de Satisfacción NTP 394 desarrollada por Pérez-Bilbao y Fidalgo) (7), en una muestra de trabajadores de un instituto especializado de rehabilitación.

\section{MATERIAL Y MÉTODOS}

Estudio transversal de tipo psicométrico, realizado en trabajadores del Instituto Nacional de Rehabilitación en el año 2013.

Se incluyeron en el estudio a trabajadores del Instituto Nacional de Rehabilitación, profesionales y administrativos. La muestra estuvo conformada inicialmente por 96 personas seleccionadas en forma no probabilística, de los cuales se excluyeron a ocho por presentar datos incompletos o inexactos, quedando finalmente constituida por 88 sujetos.

Se utilizó el instrumento de Satisfacción General de Warr, Cook y Wall traducida al español y adaptada por Pérez-Bilbao y Fidalgo, denominado Escala General de Satisfacción NTP 394 (4,7) que mide dos

Tabla 1.Características demográficas de los participantes.

\begin{tabular}{lrr}
\hline & Masculino & \multicolumn{1}{c}{ Femenino } \\
\hline Sexo & $19(21,6 \%)$ & $69(78,4 \%)$ \\
Instrucción & & \\
$\quad$ Secundaria completa & & $2(2,9 \%)$ \\
Superior técnica & $4(21,1)$ & $15(21,7 \%)$ \\
$\quad$ Superior universitaria & $15(79 \%)$ & $52(75,4 \%)$ \\
Condición Laboral & & \\
Contratado & $5(26,3 \%)$ & $18(26,1 \%)$ \\
$\quad$ Nombrado & $14(73,7 \%)$ & $51(73,9 \%)$ \\
Estado Civil & & \\
Soltero & & $24(34,8 \%)$ \\
Casado & $15(78,9 \%)$ & $35(50,7 \%)$ \\
$\quad$ Otros & $4(21,1 \%)$ & $10(14,4 \%)$ \\
Ocupación & & \\
Artesano & & $1(1,4 \%)$ \\
Técnico Administrativo & & $6(8,7 \%)$ \\
Técnico Asistencial & $3(15,8 \%)$ & $12(17,4 \%)$ \\
Cargo Directivo & $2(10,5 \%)$ & $1(1,4 \%)$ \\
Profesional asistencial & $14(73,7 \%)$ & $46(66,7 \%)$ \\
Profesional No asistencial & & $2(2,9 \%)$ \\
Sin dato & & $1(1,4 \%)$ \\
\hline
\end{tabular}


dimensiones: satisfacción intrínseca y extrínseca, basada en la teoría de los factores higiénicos de Herzberg.

La aplicación se realizó en una sola sesión de 20 minutos. Fue auto administrado en forma individual y colectiva, previa firma del consentimiento informado.

Se realizó un análisis factorial exploratorio mediante el método de ejes principales en lugar del análisis de componentes principales $(8,9)$; se eligió el método de rotación promax (10), que ha sido comprobado como el más eficiente desde sus inicios (11).

\section{RESULTADOS}

En la tabla 1, se muestran las características de los participantes.

En el análisis de los ítems se observó que, excepto los ítems 7, 10 y 11, el resto tuvo una tendencia de respuesta hacia la satisfacción moderada y satisfecha, indicando de moderada o mayor satisfacción con los contenidos muestreados (Tabla 2). La dispersión fue similar en todos los ítems, excepto el ítem 14, que mostró menos variabilidad.

La matriz de correlaciones primero fue evaluada con el coeficiente $\operatorname{KMO}(0,81)$ y la prueba de esfericidad de Barttlett (c2, [gl: 105] = 506,94, p < 0,01) indicaron que las correlaciones fueron adecuadas para analizarse por el método de análisis factorial.

En la evaluación de las dimensiones, se obtuvo claramente que un solo factor retuvo un gran monto de varianza explicada (autovalor $=5,58 ; 37,20 \%$ de la varianza total) mientras el resto de los demás potenciales factores tuvieron autovalores menores de 1,7. El gráfico scree test (12) reveló también un claro distanciamiento del primer factor respecto a los demás.

Tabla 2. Resultados del análisis factorial exploratorio, para uno y dos factores.

\begin{tabular}{|c|c|c|c|c|c|c|c|c|c|}
\hline \multirow{2}{*}{ Ítems } & \multirow{2}{*}{ M } & \multirow{2}{*}{$\mathbf{D E}$} & \multicolumn{4}{|c|}{ Solución de dos factores } & \multicolumn{3}{|c|}{ Solución de un factor } \\
\hline & & & rit & F1 & F2 &.$h^{2}$ & rit & F1 &.$h^{2}$ \\
\hline \multicolumn{10}{|c|}{ Satisfacción intrínseca } \\
\hline AIO_SL1 & 3,47 & 1,35 & 0,53 & 0,52 & 0,15 & 0,38 & 0,58 & 0,62 & 0,38 \\
\hline AIO_SL3 & 4,33 & 1,00 & 0,50 & 0,70 & $-0,09$ & 0,42 & 0,4 & 0,61 & 0,37 \\
\hline AIO_SL5 & 4,09 & 1,37 & 0,46 & 0,99 & $-0,32$ & 0,75 & 0,59 & 0,71 & 0,50 \\
\hline AIO_SL7 & 2,65 & 1,23 & 0,43 & 0,02 & 0,64 & 0,42 & 0,48 & 0,47 & 0,22 \\
\hline AIO_SL9 & 3,97 & 1,19 & 0,62 & 0,70 & 0,01 & 0,49 & 0,64 & 0,68 & 0,47 \\
\hline AIO_SL11 & 2,69 & 1,27 & 0,42 & 0,06 & 0,54 & 0,33 & 0,44 & 0,44 & 0,19 \\
\hline AIO_SL13 & 4,32 & 1,24 & 0,38 & 0,09 & 0,32 & 0,15 & 0,32 & 0,32 & 0,10 \\
\hline AIO_SL15 & 4,26 & 1,32 & 0,14 & $-0,25$ & 0,50 & 0,17 & 0,12 & 0,11 & 0,01 \\
\hline \multicolumn{10}{|c|}{ Satisfacción extrínseca } \\
\hline AIO_SL2 & 4,14 & 1,19 & 0,56 & 0,63 & 0,02 & 0,42 & 0,58 & 0,64 & 0,40 \\
\hline AIO_SL4 & 3,61 & 1,41 & 0,60 & 0,57 & 0,15 & 0,45 & 0,61 & 0,67 & 0,45 \\
\hline AIO_SL6 & 4,33 & 1,07 & 0,64 & 0,51 & 0,27 & 0,49 & 0,65 & 0,70 & 0,49 \\
\hline AIO_SL8 & 3,94 & 1,25 & 0,60 & 0,48 & 0,18 & 0,35 & 0,54 & 0,60 & 0,36 \\
\hline AIO_SL10 & 2,95 & 1,45 & 0,59 & 0,29 & 0,41 & 0,38 & 0,54 & 0,58 & 0,34 \\
\hline AIO_SL12 & 3,39 & 1,27 & 0,57 & 0,68 & 0,03 & 0,49 & 0,62 & 0,69 & 0,47 \\
\hline AIO_SL14 & 4,50 & 0,94 & 0,40 & 0,17 & 0,42 & 0,29 & 0,47 & 0,47 & 0,22 \\
\hline \multicolumn{10}{|c|}{ Correlación interfactores } \\
\hline F1 & - & - & - & 1,0 & & & - & - & \\
\hline F2 & - & - & - & 0,55 & 1,0 & & - & - & \\
\hline Alfa de Cro & & - & - & 0,73 & 0,82 & & - & 0,86 & \\
\hline
\end{tabular}

M: Media; DE: Desviación Estándar; Rit: Correlación item-test; F1: Factor 1; F2:Factor 2; h2: Comunalidades 
Para observar comparativamente la relación de los ítems con sus factores, se extrajeron dos soluciones factoriales: de uno y dos factores (Tabla 2). Se observó que el patrón de cargas factoriales en el modelo de dos factores no se ajusta de acuerdo al instrumento original; el primer factor contiene la mayoría de los ítems del instrumento, que muestran cargas más elevadas comparadas con las cargas factoriales del segundo factor. El ítem 15 muestra ser problemático para su escala original debido a su carga negativa. La correlación entre los factores es elevada, sugiriendo moderada falta de discriminación de los factores. Por otro lado, la solución de un factor presenta sus cargas factoriales alrededor de 0,45 pero el ítem 15 solo se relaciona con el factor con un bajo nivel de discriminación.

La fiabilidad por consistencia interna por el coeficiente alfa de Cronbach (13) (Tabla 2), mostraron puntajes con buenos niveles de reproducibilidad, especialmente el factor 2. También se halló que el ítem 15 continua siendo problemático, pues en el factor 1 (solución de dos factores), la fiabilidad sin el ítem 15 fue 0,76 . El puntaje total (solución de un factor) la fiabilidad sin el ítem 15 fue 0,87 , levemente mejor que cuando este ítem forma parte del puntaje.

\section{DISCUSIÓN}

Este estudio de validación aporta a los primeros resultados publicados sobre la validación de la Escala de Satisfacción General de Warr. El modelo de dos factores inicialmente propuesto (satisfacción extrínseca e intrínseca) no fue replicable en la muestra estudiada, hallándose un solo factor interpretable. El uso del instrumento, en su forma actual parece sugerir un modo más parsimonioso de representar la satisfacción laboral en la muestra de estudio. El tamaño de la muestra pone un límite en la estabilidad de los resultados y se requiere de un mayor tamaño y mayor heterogeneidad ocupacional.

Se reportan estudios con el uso de otras escalas para medir la SL tanto globales como multidimensionales. El cuestionario NTP213, validado en población española (3) y basado en la teoría de Herzberg y Crozier; así como la Escala INDSALES aplicada en el estudio de Brian Rutherford et al (14).

Si bien se encuentra en la literatura diferentes instrumentos para medir la SL, los estudios psicométricos en nuestro medio son escasos. Fernández
(15), realizó un estudio con docentes de colegios públicos de Lima, utilizando la Escala de SL de Warr correlacionando con otras variables psicosociales. Asimismo, Bobbio y Ramos (16), evaluaron la SL en personal asistencial $(n=227)$ en un Hospital de Lima, basado en una escala diseñada y validada por juicio de expertos, reportando un nivel de confiabilidad Alfa de 0,70. Palma (17) elaboró y validó una Escala de Satisfacción Laboral en empleados dependientes de Lima Metropolitana $(n=952)$ encontrando adecuadas propiedades psicométricas.

En conclusión, los resultados encontrados permiten afirmar que la Escala de Warr es válida y confiable pero sólo para una dimensión, pero, hay que tener en cuenta que el estudio tuvo como limitaciones una muestra reducida y procedente de una sola institución.

\section{Agradecimiento:}

A la Dra. Carmen Tataje Contreras, Jefa de la Oficina de Apoyo a la Investigación y Docencia

Especializada del INR, por su invalorable apoyo.

\section{Declaración de Financiamiento y de conflictos de intereses:}

No existe conflicto de intereses

\section{Correspondencia:}

Alicia Herminia Boluarte Carbajal. Jr. Julio Rodavero 890, Pueblo Libre. Correo electrónico: aliciaboluarte@gmail.com.

\section{REFERENCIAS BIBLIOGRÁFICAS}

1. Schultz D. Psicología Industrial. $3^{\mathrm{a}}$ edición. México DF: McGraw-Hill; 1991. p. 258.

2. Oficina Internacional del Trabajo. El diálogo social en los servicios de salud: instituciones, capacidad y eficacia. Organización Internacional del trabajo. Ginebra: Oficina Internacional del Trabajo; 2002.

3. Meliá J, Peiró J. La medida de la satisfacción laboral en contextos organizacionales: El Cuestionario de Satisfacción S20/23. Psicologemas. 1989; 5:59-74.

4. Warr P, Cook J, Wall T. Scales for the measurement of some work attitudes and aspects of psychological well-being. Journal of Occupational Psychology. 1979; 52: 129-48.

5. Hills D, Joyce C, Humphreys J. Validation of a job satisfaction scale in the Australian Clinical. Evaluation \& the Health Professions. 2011; 35: 4776. 
6. Goetz K, Campbell S, Bjoern Broge J, Willms S, Szecsenyi, J. Evaluation of job satisfaction of practice staff and general practitioners: an exploratory study. BMC Family Practice. 2011; 12: 137.

7. Pérez-Bilbao J, Fidalgo M. Job satisfaction: Overall job satisfaction scale. Barcelona: Centro Nacional de Condiciones de Trabajo; 1995.

8. Fabrigar L, Wegener D, MacCallum R, Strahan, E. Evaluating the use of exploratory factor analysis in psychological research. Psychological Methods. 1999; 4:272-299.

9. Snook S, Gorsuch R. Component analysis versus common factor analysis: A Monte Carlo study. Psychological Bulletin. 1989; 106:148-154.

10. Hendrickson A, White P. Promax: a quick method for rotation to oblique simple structure. British Journal of Statistical Psychology. 1964; 17:65-70.

11. Gorsuch R. A comparison of biquartimin, maxplane, promax, and varimax. Educational and Psychological Measurement. 1970; 30:861-872.

12. Cattell R. The scree test for the number of factors. Multivariate Behavioral Research. 1966; 1:245-276.

13. Cronbach L. Coefficient alpha and the internal structure of tests. Psychometrika.1951: 16, 297-334.

14. Rutherford B, Boles J, Hamwi G, Madupalli R, Rutherford L. El papel de las siete dimensiones de la satisfacción laboral en las actitudes y comportamientos del vendedor. Journal of Business Research. 2009; 62 (11): 1146-1151.

15. Fernández M. Burnout, autoeficacia y estrés en maestros peruanos: Tres estudios fácticos. Ciencia \& Trabajo. 2008; 39 (10): 120-125.

16. Bobbio L, Ramos W. Satisfacción laboral y factores asociados en personal asistencial médico y no médico de un hospital nacional de Lima-Perú. Revista Peruana de Epidemiología. 2010:14 (2): 133-138.

17. Palma S. Elaboración y Validación de una Escala de Satisfacción Laboral SL-SPC para trabajadores de Lima Metropolitana. Santa Fe: El Cid Editor; 2009. p.16.

Recibido: 17/03/2014

Aceptado: 09/06/2014 